\title{
Repetition blindness and repetition priming: Effects of featural differences between targets and distractors on RSVP dual-target search
}

\author{
Paul E. Dux \\ Vanderbilt University, Nashville, Tennessee \\ AND \\ VERonika COLTheart \\ Macquarie University, Sydney, Australia
}

\begin{abstract}
In six experiments, we investigated the influence of featural differences between targets and distractors on the detection and identification of dissimilar and repeated targets in conditions that typically produce an attentional blink and repetition blindness (when a target is repeated). Rapid serial visual presentation streams were presented that contained letter targets and distractors that were either letters or digits. The targets and the distractors were either the same color or a different color and were presented either in the same font or in a different font. Dual-target performance on nonrepeat trials was strongly enhanced when the targets were colored. In addition, when subjects used either the color or the font cues to select the targets, there was benefit of repetition on dualtarget report, instead of repetition blindness. The results suggest that featural differences between targets and distractors play an important role in registering stimuli as distinct objects.
\end{abstract}

In rapid serial visual presentation (RSVP), stimuli appear one after another in the same spatial location for approximately $100 \mathrm{msec}$ each (Potter \& Levy, 1969). Research in which this paradigm has been used has identified two fundamental limitations of conscious visual perception: the attentional blink (Broadbent \& Broadbent, 1987; Raymond, Shapiro, \& Arnell, 1992; Weichselgartner \& Sperling, 1987) and repetition blindness (Chun, 1997; Johnston, Hochhaus, \& Ruthruff, 2002; Kanwisher, 1987). The attentional blink refers to subjects' impaired ability to report the second of two different targets in an RSVP stream if it appears within 200-600 msec of Target 1 (T1). Repetition blindness is an additional deficit characterized by a subject's inability to report both instances of a repeated target if they are presented within $500 \mathrm{msec}$ of one another.

The attentional blink and repetition blindness share many similarities, including the conditions (RSVP) under which they are observed and their approximate time courses ( $\sim 500 \mathrm{msec})$; however, these two processing deficits can be experimentally dissociated (Chun, 1997; see also Dux $\&$ Marois, 2007). Chun demonstrated that the attentional blink was completely removed when letter targets were presented among computer symbol distractors, as opposed to digit distractors; however, this manipulation did not affect repetition blindness. Conversely, repetition blindness was removed when red and green target letters appeared among black letter distractors, whereas a significant attentional blink was still observed under these conditions. To account for these results, Chun hypothesized that the two deficits represented distinct bottlenecks of information processing, one limiting the rate at which stimuli can be recognized (attentional blink), and the other restricting the rate at which items can be registered as separate stimuli (repetition blindness). Thus, he concluded, the attentional blink was affected by target-distractor discriminability because this influenced the ease with which targets were identified, whereas repetition blindness was dependent on the episodic distinctiveness of targets because this affected the extent to which stimuli were registered as distinct objects.

Chun's (1997) data clearly demonstrate that the attentional blink is influenced by categorical differences between targets and distractors, which Dux and Coltheart (2005) have demonstrated is due to changes in the levels of both perceptual and conceptual masking. Thus, in RSVP, target identification appears to be strongly influenced by the extent to which distractors interfere with targets. However, what role does target-distractor discriminability play in the registration of stimuli as distinct items? Put differently, are there differences between targets and distractors that can influence repetition blindness? From Chun's study, it appears that categorical differences between targets and distractors do not influence repetition blindness; however, what is not known is whether featural differences between targets and distractors can aid stimulus registration. Chun's finding that repetition blindness did not occur when targets differed in color from each other and

P.E.Dux, paul.dux@vanderbilt.edu 
from the distractors does not resolve this issue, since featural differences between the two targets and the distractors were confounded in this experiment. Here, we tested whether such differences between targets and distractors alone are sufficient to prevent the occurrence of repetition blindness, with a view to understanding how differences between task-relevant and task-irrelevant stimuli influence target registration in visual processing.

Two studies have previously examined the effect of featural differences between targets and distractors on repetition blindness. Campbell, Fugelsang, and Hernberg (2002) presented RSVP sentences in which the targets were either words or nonwords and, at the end of each trial, subjects were presented with three items and were required to report the frequency of each in the stream (zero, once, or twice). On half the trials, the targets were brighter than the distractors and were colored yellow rather than white. Reduced, but still significant, repetition blindness occurred when the word targets and the distractors were featurally different, suggesting that such differences between targets and distractors may enhance target registration but are not sufficient to remove repetition blindness. Similarly, Kanwisher (1991) found robust repetition blindness when subjects were presented with RSVP streams of letters, where the targets always had a color different from that of the distractors. Significant repetition blindness was found between nominally identical targets that had the same color and those that differed in color, and repetition blindness magnitude did not differ between these conditions.

The results discussed above indicate that whereas featural differences between targets are sufficient to prevent the occurrence of repetition blindness, such differences between targets and distractors are not. However, further research examining the influence of featural differences between targets and distractors on target registration is warranted for a number of reasons. Chun (1997) demonstrated that the color differences between the two targets, as well as the distractors, prevented the occurrence of repetition blindness only when a large number of trials were presented. In his experiment, approximately 280 trials were used, and the overall result was a null effect of target repetition, with a trend toward repetition priming. An examination of the early blocks of this experiment showed that subjects initially demonstrated repetition blindness, suggesting that they had to learn to use the featural cues to distinguish the repeated items. Therefore, Kanwisher (1991) may have failed to find a reduction in repetition blindness when the targets had the same color because she presented only 36 trials. In addition, in Campbell et al.'s (2002) study, featurally defined and nonfeaturally-defined target trials were intermixed within blocks. Under these conditions, subjects were unable to rely on featural differences to serve as episodic cues for the targets in every trial, and as a result, repetition blindness may have still been observed because the subjects did not employ the featural cues to select targets. Here, we aimed to investigate whether featural differences between targets and distractors are, indeed, sufficient to enhance the registration of stimuli and prevent the occurrence of repetition blindness in dual-target RSVP search. Subjects were presented a large number of trials, and featural target cues were kept constant within blocks of trials.

\section{EXPERIMENT 1}

In Experiment 1, we investigated whether repetition blindness would occur for identical targets that differed in color from distractors. ${ }^{1}$ Two capital letter targets, either red or black, were presented within a stream of black digit distractors, the targets being either identical (e.g., $P, P$ ) or different (e.g., $P, D$ ). Chun (1997) hypothesized that "color cues allow for targets to pop out, providing two salient spatiotemporal tokens that may serve as episodic anchors for object token instantiation" (p. 752). Therefore, if featural differences between targets and distractors can capture attention and enhance registration of the second target, dualtarget search may not be vulnerable to repetition blindness.

\section{Method}

Subjects. Twenty undergraduate and postgraduate students at Macquarie University took part in the experiment. The sample consisted of 6 males and 14 females, with an average age of 24.8 years, ranging from 18 to 36 years. All the subjects reported normal or corrected-to-normal visual acuity and normal color vision.

Stimulus materials and Apparatus. Stimuli were presented centrally in the same spatial location for $100 \mathrm{msec}$ each, with no interstimulus interval. The stimuli were presented in Courier New font and, at a viewing distance of approximately $40 \mathrm{~cm}$, subtended $0.7^{\circ}$ of visual angle. Red letters, black letters, and black digits appeared on a white background. The experiment was programmed in DMDX (Forster \& Forster, 2003) and was conducted using a Dell Flat Trinitron monitor with a $120-\mathrm{Hz}$ vertical refresh rate controlled by a Dell PC computer.

The targets were selected from the alphabet, excluding the letters $I, L, O, Q, U$, and $V$, and the distractors were selected from the digits $2-9$. The stimuli were presented as targets an equal number of times, with no target pair of items on nonrepeat trials appearing more than once. The distractors were randomly selected without replacement from the distractor stimulus set for each trial. Both targets were capital letters.

Trial structure. Test trials contained either two red or two black letter targets and eight black digit distractors, with a black " $\&$ " mask appearing at the end of each stream. T1 was always presented in Serial Position 3, with Target 2 (T2) following an equal number of times at lags 2-6. A minimum of two items followed T2 (a digit and the " $\&$ " mask). In half the trials, T1 and T2 had the same identity (repeat trials; e.g., $P, P$ ), and in the other half, a different identity (non repeat trials; e.g., $P, D$ ). In addition, single T1 trials were also presented to the subjects; these were identical to dual-target trials, with the exception that $\mathrm{T} 2$ was replaced with a distractor. There were two experimental blocks; in one block of trials, the targets were colored red, and in the other block, the targets were the same color as the distractors (black). Block order was counterbalanced across subjects.

Procedure. The subjects initiated each trial by pressing the space bar. Trials began with a fixation cross for $300 \mathrm{msec}$, followed by the sequence of stimuli, each being presented for $100 \mathrm{msec}$. The subjects were required to report either the red letter target( $s$ ) or the letter target (s) at the end of each trial when a prompt, "Please recall the targets," appeared in the center of the screen. It was emphasized that it was possible for the targets to have the same identity in a trial and that, if this was the case, the subjects were to report the stimulus name twice. The subjects were also informed that there were also trials on which only one target was presented.

The subjects completed 20 practice trials before each experimental block that contained two examples of each condition (rep- 


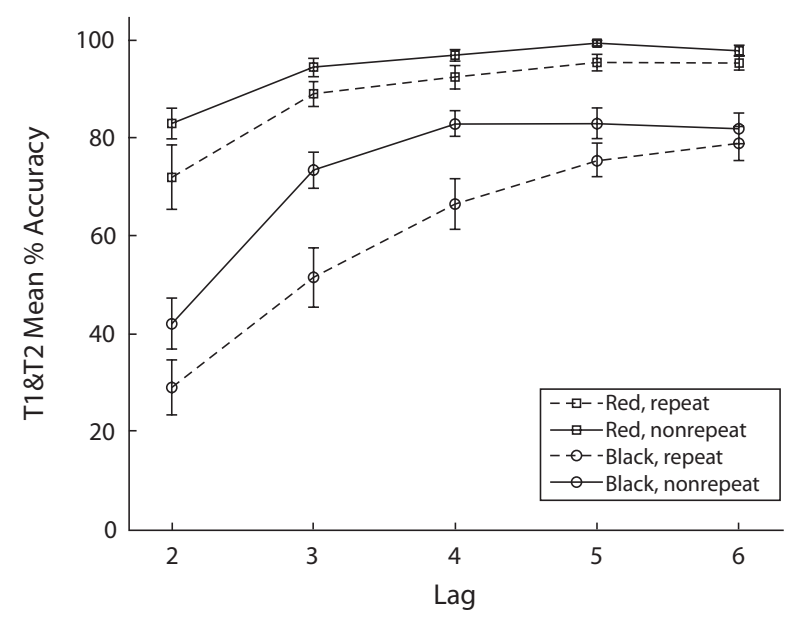

Figure 1. Mean percentage of trials in Experiment 1 where both Target 1 and Target 2 (T1\&T2) were reported correctly as a function of target color, repetition, and lag.

etition $\times$ lag). Each of the practice blocks was then followed by a block of 100 experimental trials. Each block contained 10 repeat and 10 nonrepeat dual-target trials at each lag and 25 filler trials containing one target only.

Design. This experiment had a $2 \times 2 \times 5$ repeated measures design. The first independent variable was target color, which was manipulated across block and had two levels: red and black. The second independent variable, repetition, was manipulated within each block and also had two levels: repeat and nonrepeat. The final independent variable, lag, was also manipulated within each block and had five levels: lags 2, 3, 4, 5, and 6. The lag manipulation provides an index of the attentional blink, whereas the effect of repetition and its direction indicate the presence of either repetition blindness or repetition priming. The dependent variable was target report accuracy.

\section{Results}

T1 accuracy data for the nonrepeat trials and the joint target report data - that is, the number of trials on which both T1 and T2 (T1\&T2) were reported accurately - were analyzed separately. In addition, the T1\&T2 accuracy data were also analyzed after being adjusted for guessing, although the subjects reported only a small number of repetition intrusions. A repetition intrusion occurs when a subject reports that the targets were identical on a nonrepeat trial. This type of response would have improved performance on repeat trials and reduced accuracy on nonrepeat trials, perhaps leading to inflated performance in the repeat trials. To address this potential problem, we employed the conservative guessing adjustment used by Chun (1997) for each subject at each lag. In addition, we also examined intrusions made on the single-target trials, in an attempt to assess further the possible influence of a guessing bias. All the data are reported here as percentages, and the responses were counted as correct regardless of their order of report.

Target 1 accuracy for nonrepeat trials. In this and all subsequent analyses, block order had no effect on target accuracy and did not interact with any of the other variables. As a result, none of the analyses included it as a factor. The T1 data were subjected to a $2 \times 5$ repeated measures ANOVA with the factors of target color and lag. The only significant effect was that of target color $[F(1,19)=15.6, p<.001]$, with target report being superior when the target was colored red (99.5\%), as compared with black (95.2\%). The high level of T1 accuracy, in both the red and the black target blocks, indicates that the subjects did not have difficulty reading stimuli presented in either of the font colors.

Target 1 and Target 2 accuracy. Figure 1 shows the percentage of trials on which both T1 and T2 were reported correctly as a function of target color, repetition, and lag. The T1\&T2 accuracy data were subjected to a $2 \times 2 \times 5$ repeated measures ANOVA, and Type I error was controlled by employing a Bonferroni correction.

There was a significant main effect of target color $[F(1,19)=122.3, p<.0002]$, with target report superior for red targets $(91.65 \%)$, as compared with black targets $(66.5 \%)$. The main effect of repetition $[F(1,19)=17.7, p<$ .0006] was also significant, with target report better on nonrepeat trials $(83.6 \%)$ than on repeat trials $(74.6 \%)$. A significant main effect of lag $[F(4,76)=57.9, p<.0002]$ was also found, with target report superior at later lags, as compared with early lags, indicating the presence of an attentional blink. There were three significant two-way interactions. First, target color and repetition interacted $[F(1,19)=5.8$, $p<.05]$, indicating that although there was significant repetition blindness both when the targets were red and when they were black, the effect was larger for black target trials. Second, there was a significant interaction between target color and lag $[F(4,76)=13.2, p<.0002]$. This occurred because, in the black target trials, target report was lower at lag $3(62.5 \%)$ than at lag $4(74.8 \%)[F(1,76)=17.5$, $p<.0002]$, whereas this was not the case for red targets. Finally, repetition and lag $[F(4,76)=3.2, p<.02]$ also significantly interacted, indicating that repetition blindness decreased with increasing lag.

Intrusions on dual-target trials. Out of a total of 2,000 trials in the black target condition, intrusion errors 
occurred on $8.6 \%$ of the trials. Nonrepetition intrusions on repeat trials occurred on $5 \%$ of all trials; nonrepetition intrusions on nonrepeat trials occurred on 3.3\% of all trials; and repetition intrusions on nonrepeat trials accounted for only $0.3 \%$ of all trials. In the red target condition, the subjects made intrusion errors on $2.8 \%$ of all trials: $1.55 \%$ of all trials were nonrepetition intrusions on repeat trials; $1.1 \%$ of all trials were nonrepetition intrusions on nonrepeat trials; and $0.15 \%$ of all trials were repetition intrusions on nonrepeat trials.

Intrusions on single-target trials. A single-target trial response was counted as incorrect if the wrong letter was reported or if more than one item was reported. The subjects made intrusion errors on 96 (19.2\% of all the trials) of the 500 filler trials in the black target condition: $14 \%$ of all trials were correct answers on which the subjects also recalled a second different letter; $2.8 \%$ of all trials were correct answers on which the subjects also reported a repeat; $1.8 \%$ of all trials were reports of a single incorrect letter; and finally, only $0.6 \%$ of all trials were reports of two incorrect letters. In the red target block, only three intrusions occurred in the 500 single-target trials presented.

\section{Discussion}

In both the red and the black target blocks, a typical attentional blink pattern was found for the nonrepeat trials, with joint target recall being lowest at lag 2 and gradually improving as lag increased. The attentional blink was strongly influenced by target color, with the deficit significantly reduced for red target trials, as compared with black target trials. This result suggests that the colored stimuli captured attention and, as a result, enhanced target identification. Significant repetition blindness was also present in both blocks, and as was the case with the attentional blink, the effect appeared to be attenuated on trials on which the targets were red rather than black. However, this reduction in repetition blindness could also be due to a ceiling effect. This hypothesis is supported by there being no significant difference in repetition blindness magnitude at lag 2 (the lag at which red target nonrepeat accuracy was not at ceiling) between black and red target trials $(F<1)$. In addition, when the T1\&T2 data were subjected to a logistic transformation and tested with the same ANOVA as above, there were only significant effects of target color and lag $(F$ s $>$ $33.2, p$ s $<.0001)$, and these variables did not interact with repetition $(F<1)$. Thus, the present experiment suggests that color differences do not influence target registration, supporting the previous findings of Kanwisher (1991) and Campbell et al. (2002), both of whom demonstrated significant repetition blindness when identical targets differed in color from the distractors. An examination of the intrusion errors made by the subjects, on both dual- and single-target trials, as well as the failure to find any difference in the pattern of results after the T1\&T2 data were adjusted for guessing and submitted to the same ANOVA as above, demonstrates that a bias toward reporting repeated targets did not influence the results. Similarly, Chun (1997) had found a low incidence of repeat intrusions on nonrepeat trials and found that an absence of repetition blindness was not due to a guessing bias.

\section{EXPERIMENT 2}

In Experiment 1, significant repetition blindness was observed when two red targets differed in color from the distractors. This demonstrates that featural differences between targets and distractors do not enhance target registration and prevent the occurrence of repetition blindness. However, did we find repetition blindness in the red target block because the targets were defined not only by color, but also by category? In Chun's (1997) third experiment, repetition blindness did not occur when the targets were different colors, the distractors were black, and all of the stimuli were letters. Interestingly, a trend toward repetition blindness occurred when the targets also differed in category from the distractors (Chun, 1997, Experiment 4). This suggests that repetition blindness fails to occur only when subjects use featural information as the target-defining cue.

Experiment 2 had two aims. First, we tested the hypothesis that repetition blindness is alleviated when subjects use featural information to select targets. Second, we wanted to investigate whether or not any benefit of these featural differences between targets and distractors on repetition processing is contingent on the targets' being separated by at least one distractor. If the featural differences between targets and distractors alleviate repetition blindness because they provide two distinct episodic markers for target registration, presenting the two featurally defined target stimuli adjacent to one another should elicit repetition blindness. Under these conditions, the featural cues will not be separable, and as a result, the repeated targets will be encoded as the same object (Chun \& Cavanagh, 1997).

Here, we presented RSVP streams containing red letter targets and black letter distractors; on all trials, the targets differed in case, and the distractors were a mixture of upperand lowercase stimuli. Defining targets by color ensured that the subjects used the featural cues to select targets. In addition, by presenting the targets in a different case, we were able to have the targets appear next to one another in an RSVP stream (lag 1), since they could be differentiated on the basis of shape. This allowed us to test whether or not the featural differences between targets and distractors remove repetition blindness and whether these cues need to be separated by a distractor in order for repetition blindness to be removed in a dual-target RSVP stream.

\section{Method}

The method for Experiment 2 was identical to that in the first experiment, except where specified.

Subjects. Twelve new undergraduate and postgraduate students at Macquarie University took part in the experiment. The sample consisted of 4 males and 8 females, with an average age of 22.9 years, ranging from 17 to 31 years.

Stimulus materials and Procedure. All the stimuli in Experiment 2 were letters, selected from the same set as that used for the targets in Experiment 1. Fourteen items were presented on each trial, with the targets always colored red and the distractors always black. The targets always differed in case. On half the trials, T1 was presented in uppercase and T2 in lowercase; on the other half, the case of the targets was reversed. The distractor stream contained an equal number of uppercase and lowercase stimuli that were randomly intermixed. T1 appeared at Serial Positions 3-7, and T2 appeared at lags 1-6 an equal number of times. The subjects were instructed to report the colored 


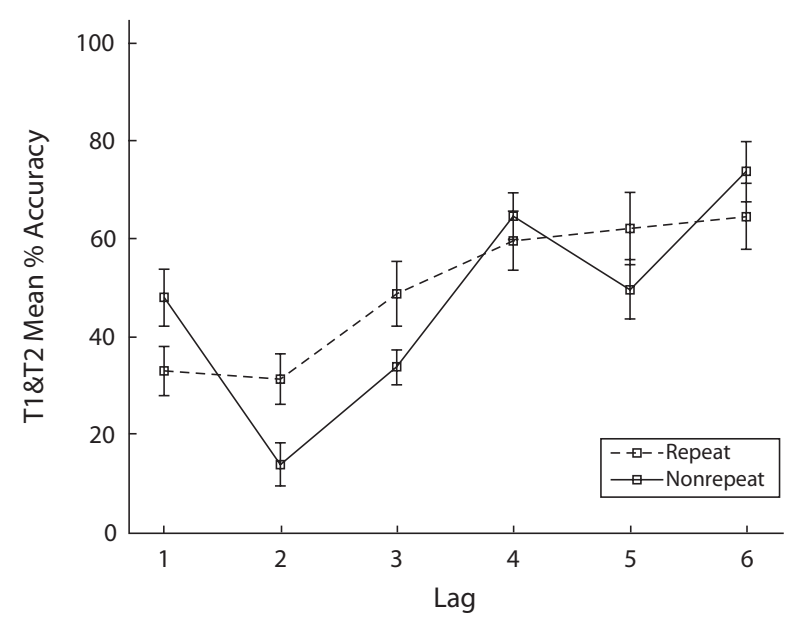

Figure 2. Mean percentage of trials in Experiment 2 where both Target 1 and Target 2 (T1\&T2) were reported correctly as a function of repetition and lag.

targets and initially completed 20 practice trials before undertaking two blocks of experimental trials, each of which contained $60(5 \times 12$ conditions) dual-target trials and 15 single-target trials.

Design. Experiment 2 had a $2 \times 6$ repeated measures design. The first independent variable was repetition, which had two levels: repeat and nonrepeat. Lag was the second independent variable and had six levels: lags 1-6. Once again, the dependent measure was the percentage of targets reported accurately.

\section{Results}

Target 1 accuracy for nonrepeat trials. Overall, T1 accuracy was $84.4 \%$. T1 accuracy data were subjected to a repeated measures ANOVA with the single factor of lag. There was a significant main effect $[F(5,55)=4.9, p<$ $.001]$, with target report being inferior at lag $2(72.5 \%)$ relative to that at lags $3-6(85 \%)[F(1,55)=10.87, p<$ $.002]$ and superior at lag $1(94.2 \%)$ relative to lags $3-6$ $[F(1,55)=5.9, p<.02]$.

Target 1 and Target 2 accuracy. Figure 2 shows the percentage of trials on which both $\mathrm{T} 1$ and $\mathrm{T} 2$ were reported correctly as a function of repetition and lag. The T1\&T2 accuracy data were subjected to a $2 \times 6$ repeated measures ANOVA.

The main effect of lag was significant $[F(5,55)=21.8$, $p<.0002]$. In addition, this variable significantly interacted with repetition $[F(5,55)=6.9, p<.0002]$. Followup tests showed there to be superior target report for repeat trials, as compared with nonrepeat trials, at lags 2 $[F(1,55)=10.8, p<.002]$ and $3[F(1,55)=7.9, p<$ $.01]$ and inferior report for repeat trials, as compared with nonrepeat trials, at lag $1[F(1,55)=7.92, p<.01]$. The main effect of repetition was not significant.

Intrusions on dual-target trials. Across the 1,440 dual-target trials in the experiment, the subjects made intrusion errors on 534 occasions (37.1\% of all trials). Nonrepetition intrusions in the nonrepeat condition occurred on $20.3 \%$ of all trials; nonrepetition intrusions on repeat trials accounted for $16.4 \%$ of all trials; and repetition intrusions on nonrepeat trials accounted for only $0.4 \%$ of all trials.
This large number of intrusions, relative to that observed in the red target block in Experiment 1, was most likely due to the increased likelihood, in the present condition, that illusory conjunctions (binding the color red to the wrong item) would occur, since the targets and the distractors were drawn from the same category (the subjects could report a red letter but would be unlikely to report a red digit).

Intrusions on single-target trials. The subjects made intrusion errors on only 46 (12.8\% of all trials) of the 360 filler trials. On $8.9 \%$ of all trials, the subjects reported a single incorrect letter; on $3.3 \%$ of all trials, the correct letter was recalled with another, different letter; and finally, on only $0.6 \%$ of all trials, the target letter was reported correctly, and another, identical item was also recalled.

\section{Discussion}

As in Experiment 1, joint target report indicated the presence of an attentional blink in the nonrepeat trials, since performance was lowest at lags 2 and 3 and improved with increasing lag. Performance was also relatively good at lag 1 on nonrepeat trials, demonstrating lag 1 sparing, another characteristic of the attentional blink (see Visser, Bischof, \& Di Lollo, 1999). Contrary to the results of Experiment 1 , a comparison of repeat and nonrepeat trials across lags 2 and 3 (the lags at which repetition blindness is typically maximal) revealed no repetition blindness; in fact, the subjects performed better on repeat trials than on nonrepeat trials (repetition priming). In addition, the opposite pattern of results was observed at lag 1, with significant repetition blindness observed in this condition. An analysis of the intrusion errors made by the subjects, on both single- and dual-target trials, showed that this result was not due to a bias toward guessing repeated targets. This was confirmed when the T1\&T2 data were reanalyzed after having been adjusted for this potential guessing bias.

Experiment 2 had two aims: to test whether repetition blindness is alleviated in dual-target RSVP search when 
subjects use featural cues to select targets and to examine the effect of repetition when two featurally defined targets are presented adjacent to one another in the RSVP stream. The results at lags 2 and 3 in this experiment indicate that when targets are the same color but differ in color from distractors, repetition blindness does not occur, and target repetition benefits dual-target report. ${ }^{2}$ These results differ from those reported by Kanwisher (1991) and Campbell et al. (2002). In Experiment 1, repetition blindness was still present when the targets were both featurally and categorically defined, a task in which the subjects did not have to employ featural information in order to select the targets. Here, we made the distractors the same category as the targets, thus maximizing the salience of the featural cue, since this was the only attribute that the subjects could use to select the targets. Therefore, it appears that featural differences between targets and distractors can prevent the occurrence of repetition blindness in an RSVP stream, if subjects use these featural cues for target selection. Interestingly, the data suggest that subjects do not always use featural information to select targets (when it is present). Perhaps, subjects typically rely more on categorical cues for selection, since this reduces the likelihood of experiencing interference from illusory conjunctions.

Interestingly, a large repetition blindness effect was present at lag 1 . This was the only lag position for which it could be argued that there was only one abrupt color onset instead of two, since at lag 1 the two red stimuli appeared next to one another and were not separated by a black distractor stimulus. Thus, it appears that two distinct separable featural cues in the stream are required before target registration can be enhanced and an improvement in performance on repeat trials can occur. It should also be noted that there was a large T $1 \& \mathrm{~T} 2$ accuracy difference between the nonrepeat trials in this experiment and those in Experiment 1; issues relating to overall performance differences between experiments will be discussed later.

\section{EXPERIMENT 3}

Experiment 2 demonstrated that repetition priming is elicited in RSVP streams when repeat targets are defined by identical color cues. This effect appears to be contingent on the two color markers being episodically separate from one another, because repetition blindness was observed in the last experiment when the colored targets were adjacent. If the repetition blindness found at lag 1 in Experiment 2 occurred because there was only a single abrupt color onset, repetition should benefit target report at lag 1 when targets are made featurally distinct not only from the distractors, but also from each other. Here, the subjects were presented with streams identical to those used in Experiment 2; however, T1 was now colored green rather than red. Thus, when targets appeared adjacent in the stream, there were two abrupt color onsets instead of one - one green and one red. We predicted that repetition priming should be elicited at lag 1 under these conditions, since the subjects could now discriminate the two target stimuli from one another on the basis of color.

\section{Method}

The method for Experiment 3 was identical to that in the second experiment, except where specified.

Subjects. Eight new undergraduate students at Macquarie University took part in the experiment. The sample consisted of 1 male and 7 females, with an average age of 21.6 years, ranging from 18 to 27 years.

Stimulus materials and Procedure. T1 was colored green, T2 red, and the distractors black. The stimuli appeared on a uniform gray field.

\section{Results}

Target 1 accuracy for nonrepeat trials. Overall, T1 accuracy was $81.9 \%$. T1 accuracy data were submitted to a repeated measures ANOVA with the single factor of lag. The analysis yielded a nonsignificant effect $(F<1)$.

Target 1 and Target 2 accuracy. Figure 3 plots the mean percentage of trials on which both the first and the second targets were reported correctly as a function of repetition and lag. The T1\&T2 accuracy data were submitted to a $2 \times 6$ repeated measure ANOVA with repetition and lag as factors.

There was a significant main effect of repetition $[F(1,7)=17.7, p<.0005]$, with greater accuracy on repeat trials $(53.8 \%)$ than on nonrepeat trials $(43.5 \%)$. The main effect of lag $[F(5,35)=26.7, p<.0002]$ was also significant. The interaction between repetition and lag was not significant.

Intrusions on dual-target trials. In Experiment 3, the subjects made intrusion errors on 371 (38.6\% of all trials) of the 960 dual-target trials presented: $19.1 \%$ of all trials were nonrepetition intrusions on nonrepeat trials; $18.5 \%$ of all trials were nonrepetition intrusions on repeat trials; and $1 \%$ of all trials were repetition intrusions on nonrepeat trials.

Intrusions on single-target trials. Across the 240 single-target trials in the experiment, the subjects made intrusion errors on only 32 ( $13.3 \%$ of all trials) occasions: $12.5 \%$ of all trials were those on which the subjects reported a single incorrect letter, and the remaining errors $(0.8 \%$ of all trials) were those on which the subjects recalled the target but also another, different letter. Thus, the subjects never reported a repeat letter in the single-target trials in this experiment.

\section{Discussion}

Once again, a typical attentional blink pattern of data was found for the nonrepeat trials, with joint target performance lowest at lags 2 and 3 and gradually improving as lag increased. In addition, lag 1 sparing was again present, since the subjects' joint target accuracy at lag 1 was better than that at both lags 2 and 3. Of greatest interest in this experiment was the interaction effect between repetition and lag. In Experiment 2, we found a significant interaction between these two variables, with repetition priming elicited at lags 2 and 3 and repetition blindness at lag 1 . In the present experiment, there was no interaction between repetition and lag, and instead, a main effect of repetition was observed. The subjects performed better on repeat trials than on nonrepeat trials, and this was particularly the case at lags 1 and 2. Thus, it appears that repetition blind- 


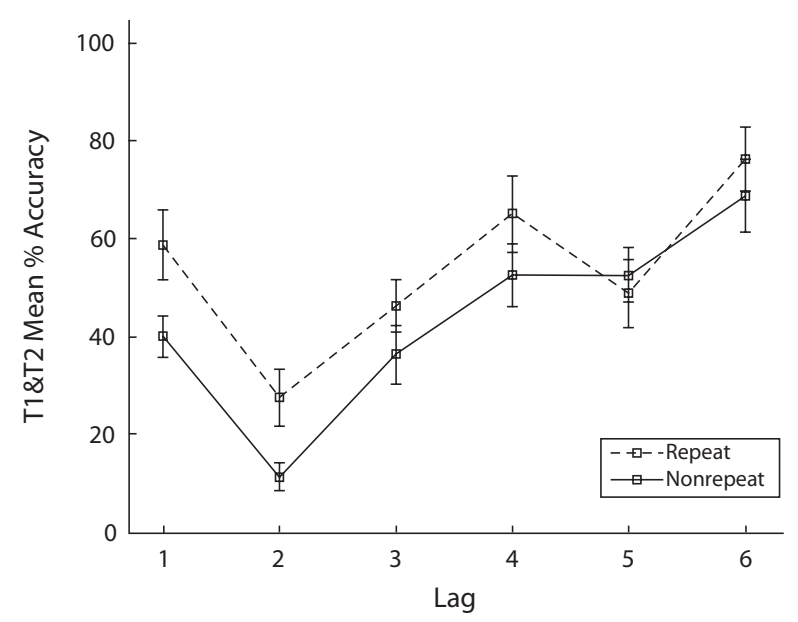

Figure 3. Mean percentage of trials in Experiment 3 where both Target 1 and Target 2 (T1\&T2) were reported correctly as a function of repetition and lag.

ness occurred in the previous experiment, when the targets were adjacent, because the subjects were unable to use the identical color cues to distinguish the targets. Here, the targets were a different color not only from the distractors, but also from each other, resulting in two abrupt color onsets at lag 1 . As a result, the subjects were able to use these cues as episodic markers for attention, and consequently, repetition benefited target report. This finding cannot be attributed to guessing, since once again the subjects made few repetition guesses and the guessing adjustment applied to the data had no effect on the overall pattern of results.

It should be noted that our results are different from those reported by Chun (1997), even though we used a methodology very similar to the one he employed. Chun found only a trend toward repetition priming, whereas we found a significant benefit of repetition on joint target report. This difference could be due to the fact that Chun varied target color ( $\mathrm{T} 1$ could be green and T2 red, or vice versa), whereas we held it constant ( $\mathrm{T} 1$ was always green, and $\mathrm{T} 2$ always red).

\section{EXPERIMENT 4}

Thus far, we have demonstrated that repetition priming occurs in dual-target RSVP streams when the targets differ featurally from the distractors. We have suggested that these featural differences act as spatiotemporal cues for attention and that this enhances the encoding of the targets on repeat trials. This effect appears to be contingent on the targets' being separated by at least one distractor or being different featurally from one another. These findings suggest that the two cues must be episodically distinct in order for the two repeat stimuli to be seen as different objects and for there to be a benefit of repetition on target report. A problem for this hypothesis is that we failed to find repetition priming and, instead, found repetition blindness in Experiment 1 when the targets were red letters among black digit distractors. We have suggested that this occurred because the subjects used categorical information, rather than the featural cues, to select the targets (the fact that the targets were letters among digits, instead of red stimuli among black stimuli). However, this hypothesis remains to be tested empirically.

If the repetition blindness found in Experiment 1 was due to the subjects' attending to category information instead of the color cues, repetition priming should still be elicited in tasks in which the targets are both categorically and featurally defined, if the color information is made more attentionally salient. Making the color information more important to the task would increase the likelihood that the subjects would use this featural property for target selection, and thus, it would be more likely that registration would be enhanced on repeat trials. To test this hypothesis, we presented RSVP streams that contained red letter targets and, within each block, manipulated whether or not the distractors were black letters or digits. By randomly varying the category of the distractors and always presenting red letter targets, we ensured that the subjects could rely on category information to distinguish the targets on only half the trials, whereas the color cue was valid on all the trials. Therefore, we predicted that increasing the reliability of the color cue, relative to the categorical cue, would lead the subjects to attend more to the color information to identify the targets and, thus, repetition priming would occur on trials on which the targets were both featurally and categorically defined.

\section{Method}

The method for Experiment 4 was identical to that in the second experiment, except where specified.

Subjects. Ten new subjects, undergraduates at Macquarie University, took part in the experiment. The sample consisted of 4 males and 6 females, with an average age of 20.1 years, ranging from 18 to 28 years.

Stimulus materials and Procedure. The stimuli in Experiment 4 consisted of the 20 uppercase letters used in the previous experiments, as well as the eight digits used in Experiment 1 . The targets were uppercase letters always colored red, and the distractors 


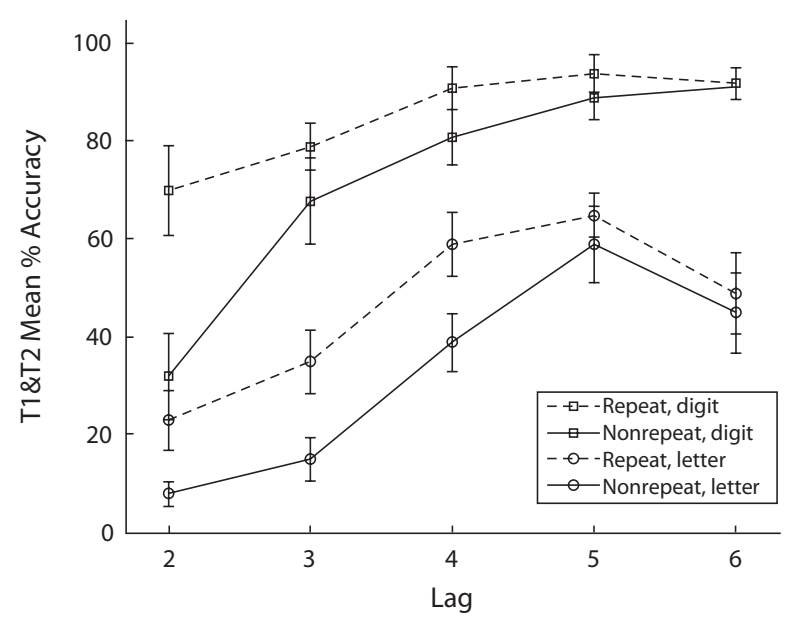

Figure 4. Mean percentage of trials in Experiment 4 where both Target 1 and Target 2 (T1\&T2) were reported correctly as a function of repetition, distractor type, and lag.

were always black. T1 position was held constant at serial Position 3, whereas T2 appeared equally often at lags $2-6$. Within each block, the distractors were either black uppercase letters or black digits. The subjects initially completed 20 practice trials and then undertook two blocks of 100 test trials each $(5 \times 20$ conditions). In addition to the dual-target trials, 26 single-target trials were included in each block.

Design. Experiment 4 had a $2 \times 2 \times 5$ repeated measures design. The first independent variable was repetition, which had two levels: repeat and nonrepeat. Distractor type was the second independent variable, and it also had two levels: letters and digits. Lag was the final independent variable, and it had five levels: lags $2-6$. Once again, the dependent measure was the percentage of targets reported accurately.

\section{Results}

Target 1 accuracy for nonrepeat trials. The $\mathrm{T} 1 \mathrm{ac}-$ curacy data were submitted to a $2 \times 5$ repeated measures ANOVA. The main effect of distractor type $[F(1,9)=34.5$, $p<.0003$ ] was significant, with superior performance on trials where the distractors were digits $(97.2 \%)$ rather than letters $(76.4 \%)$. The main effect of lag $[F(4,36)=3.1, p<$ $.03]$ was also significant, with target report being greater across lags 3-6 (87.9\%), as compared with that at lag 2 $(82.5 \%)[F(1,36)=8.6, p<.006]$. The interaction between distractor type and lag was not significant.

Target 1 and Target 2 accuracy. Figure 4 plots the mean T1\&T2 accuracy as a function of repetition, distractor type, and lag. The data were submitted to a $2 \times 2 \times 5$ repeated measures ANOVA. The main effect of repetition was significant $[F(1,9)=9.9, p<.02]$, with better report of repeated targets $(65.7 \%)$ than of nonrepeated targets $(52.8 \%)$. Distractor type $[F(1,9)=206.3, p<.0002]$ also had a significant effect. The subjects were more likely to recall both the targets when the distractors were digits (78.8\%), rather than when they were letters $(39.7 \%)$. The main effect of lag $[F(4,36)=60.8, p<.0002]$ was also significant, with performance superior at later lags.

In addition to these main effects, there was a significant two-way interaction between repetition and lag $[F(4,36)=$ $7.5, p<.0003]$ and a significant three-way interaction be- tween repetition, distractor type, and $\operatorname{lag}[F(4,36)=2.9, p<$ $.05]$. The two-way interaction resulted because repetition benefited target report only at lags 2,3 , and $4\left(F_{\mathrm{s}}>18.4\right.$, $p$ s <.0002); at lags 5 and 6 , there was a null effect of repetition. The three-way interaction occurred because significant repetition priming was seen at lags 2,3 , and $4(F$ s $>7.1$, $p \mathrm{~s}<.02)$ when the distractors were letters, but only at lag 2 $[F(1,36)=45.7, p<.0002]$ when the distractors were digits. However, this three-way interaction may have resulted due to a ceiling effect in the digit distractor condition.

Intrusions on dual-target trials. Across the 1,000 dual-target trials presented in the letter distractor condition, 359 (35.9\% of all trials) responses were intrusions: $20 \%$ of all trials were nonrepetition intrusions on nonrepeat trials; $15.5 \%$ of all trials were nonrepetition intrusions on repeat trials; and $0.4 \%$ of all trials were repetition intrusions on nonrepeat trials. In the digit distractor condition, $9.1 \%$ of the 1,000 trials were intrusions: Nonrepetition intrusions on nonrepeat trials made up $4.7 \%$ of all trials; nonrepetition intrusions on repeat trials made up $2.2 \%$ of all trials; and repetition intrusions on nonrepeat trials made up $2.2 \%$ of all trials.

Single-target trial intrusions. When the distractors were letters, intrusions occurred on $17.7 \%$ of the 260 filler trials: $12.7 \%$ of all trials were errors where a single incorrect letter was reported; $4.2 \%$ of all trials were of the type in which the correct letter was reported along with a second, different letter; and $0.8 \%$ of all trials were of the type in which two different incorrect letters were reported. Only eight (3.1\% of all trials) intrusion errors occurred on the single-target trials in the digit distractor condition, all of which were reports of a single incorrect letter.

\section{Discussion}

As in the previous experiments, an examination of the joint target report in the nonrepeat trials demonstrated the presence of an attentional blink, since performance was low at lags 2 and 3 and improved with increasing lag. The sub- 


\title{
$A B C D E g$ \& $H$ \& $M \cap P a \& g W x g$ A B C D EF G H J K M N P R S T WXYZ
}

\author{
Figure 5. Targets and distractors used in Experiment 5. Distractors presented in \\ Script font appear on the top row, and targets presented in Courier New font appear \\ on the bottom row.
}

jects performed better on digit distractor trials than on letter distractor trials. This is not surprising, given that reduced dual-target accuracy is typically observed when targets and distractors are drawn from the same stimulus category (Chun, 1997). When performance was compared between repeat and nonrepeat trials in the letter distractor condition, the results resembled those of Experiment 2, in that the presentation of two identical red letter targets among a stream of black letter distractors elicited repetition priming. Of greater interest, however, was the effect repetition had on trials on which the distractors were digits. In Experiment 1, we found significant repetition blindness when two red letter targets appeared among a stream of black digit distractors. We argued that repetition priming was not found under these conditions because the subjects did not employ the color cues and, rather, used category information to select the targets. To test this hypothesis, we increased the validity of the color cue, relative to the category cue, by holding the color of the targets constant and randomly varying the distractor category. We anticipated that this increase in the predictability of the color cue would make the subjects more likely to use it to select targets and, as a result, repetition priming would arise. The results of Experiment 4 confirmed this prediction, since we found significant repetition priming when red letter targets appeared among black digit distractors. These data provide additional support to the claim that featural differences between targets and distractors are sufficient to prevent the occurrence of repetition blindness and elicit repetition priming. In addition, they demonstrate that subjects must use featural cues for target selection if repetition is to benefit report. Since the conservative guessing adjustment we applied did not influence the overall pattern of results, we can conclude that the repetition priming found here is not due to a guessing bias.

\section{EXPERIMENT 5}

We have argued, on the basis of the experiments presented above, that featural target cues prevent the occurrence of repetition blindness because they provide episodic markers for attention that facilitate target registration. Thus far, we have only employed color cues to featurally define targets, and as a result, at present, it cannot be determined whether our results generalize to other types of featural cues. To test this, here we defined targets by font type, with targets and distractors differing featurally but not categorically (see Figure 5). If repetition priming is observed under these conditions, it will provide support for our hypothesis that featural differences between target and distractors, rather than just color differences alone, enhance target registration in RSVP.

\section{Method}

The method for Experiment 5 was identical to that in Experiment 2, except where specified.

Subjects. Twelve new subjects, undergraduates and postgraduates at Vanderbilt University, took part in the experiment. The sample consisted of 4 males and 8 females, with an average age of 20.5 years, ranging from 18 to 28 years.

Stimulus materials and Procedure. All the stimuli in Experiment 5 were uppercase letters, selected from the same set as that used in the previous experiments. Thirteen items were presented on each trial. All of the stimuli were colored black, with the targets presented in Courier New font and the distractors in Script font (stimuli that differed featurally but not conceptually; see Figure 5). T1 appeared at Serial Position 5 and T2 at lags 2-6 an equal number of times. The subjects were instructed to report the stimuli presented in Courier New font (a diagram displaying target and distractor font was left with the subjects during testing) and initially completed 22 practice trials before undertaking one block that contained 100 $(10 \times 10$ conditions $)$ experimental trials and 10 filler trials. Each stimulus was presented for $120 \mathrm{msec}$ (exposure duration was increased, relative to that in previous experiments, in order to equate overall accuracy) on a Dell Pentium 4 PC computer. The experiment was programmed in MATLAB, using the Psychophysics Toolbox extension (Brainard, 1997; Pelli, 1997).

\section{Results}

Target 1 accuracy for nonrepeat trials. Overall, T1 accuracy was $84.7 \%$. The data were subjected to a oneway repeated measures ANOVA with lag as the factor. T1 accuracy did not significantly differ across lag.

Target 1 and Target 2 accuracy. Figure 6 shows the mean T1\&T2 percentage of accuracy as a function of repetition and lag for Experiment 5. The data were subjected to a $2 \times 5$ repeated measures ANOVA.

There were significant main effects of both repetition $[F(1,11)=6, p<.05]$ and lag $[F(1,11)=20.5, p<$ $.0002]$. Target report was superior on repeat trials (67.3), relative to nonrepeat trials (57.3), and improved as lag increased. The two variables did not interact.

Intrusions on dual-target trials. In Experiment 5, the subjects made intrusion errors on 224 (18.6\% of all trials) of the 1,200 dual-target trials: Nonrepetition intrusions on nonrepeat trials made up $10.9 \%$ of all trials; nonrepetition intrusions on repeat trials accounted for $7.4 \%$ of all trials; and repetition intrusions on nonrepeat trials accounted for only $0.3 \%$ of all trials.

Intrusions on single-target trials. Intrusion errors were made on 16 (13.3\% of all trials) of the 120 filler trials: $8.3 \%$ of all trials were correct answers where the subject also recalled another, different letter; $2.5 \%$ of all trials were single incorrect letter reports; and finally, only $0.8 \%$ of all trials were errors where the letter was reported correctly and another, identical item was also recalled. In addition to these errors, there were also $2(1.7 \%$ of all 


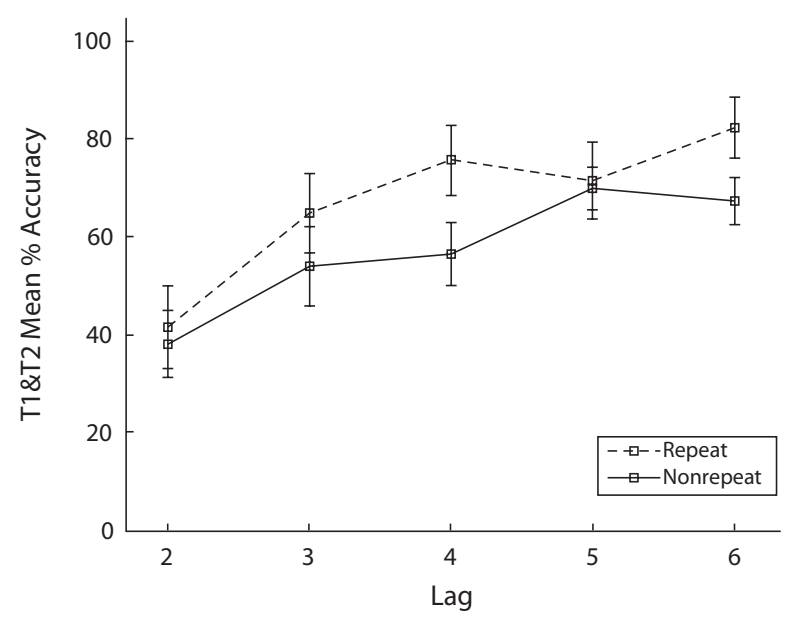

Figure 6. Mean percentage of trials in Experiment 5 where both Target 1 and Target 2 (T1\&T2) were reported correctly as a function of repetition and lag.

trials) trials on which two different targets were recalled, neither of which had appeared as targets in the stream. Thus, on single-target trials, only one repetition intrusion occurred throughout the entire experiment.

\section{Discussion}

As was the case in previous experiments, in the nonrepeat trials, there was a significant attentional blink pattern, with performance lowest at lag 2 and improving with increasing lag. In addition, overall repetition priming was observed, and at no lag was there any evidence of repetition blindness. Thus, it appears that a range of featural differences between targets and distractors, rather than simply color alone, can enhance target registration and prevent the occurrence of repetition blindness. Again, the subjects made few repetition intrusion errors, and the conservative guessing adjustment we applied to the T1\&T2 data did not influence the overall pattern of results; therefore, the repetition priming found here was not due to a guessing bias.

\section{EXPERIMENT 6}

As was previously discussed, Kanwisher (1991) found significant repetition blindness in an experiment in which targets were drawn from the same category as distractors but differed in color, a result contrary to the findings of Experiments 2-5. We have argued, along with Chun (1997), that this is due to the small number of trials $(<40)$ employed by Kanwisher (1991); however, this remains to be tested under the present conditions. Here, we presented subjects with RSVP trials containing black letter distractors and red letter targets and examined the effect of repetition every 20 trials (trial epochs). If Kanwisher's (1991) result was, indeed, due to the low number of trials employed, we should observe a reduction in repetition blindness as the number of trials increases.

\section{Method}

The method in Experiment 6 was identical to that in Experiment 2, except where specified.

Subjects. Eighteen new subjects, undergraduates and postgraduates at Vanderbilt University, took part in the experiment. The sample consisted of 5 males and 13 females, with an average age of 21 years, ranging from 18 to 31 years.

Stimulus materials and Procedure. Thirteen items were presented in each trial. All the stimuli were uppercase letters, and the targets were colored red and the distractors black. T1 appeared at Serial Position 5, and T2 at lag 2. The subjects were instructed to report the red letter stimuli and completed one block of trials that contained 100 $(50 \times 2$ conditions) experimental trials and 10 filler trials. As was the case in Kanwisher's (1991) experiment, no practice was given to the subjects. Each stimulus was presented for $106 \mathrm{msec}$ on a Dell Pentium 4 PC computer, and the experiment was programmed in MATLAB, using the Psychophysics Toolbox extension (Brainard, 1997; Pelli, 1997).

Design. Experiment 6 had a $2 \times 5$ repeated measures design. The first independent variable was repetition, which had two levels: repeat and nonrepeat. Trial epoch was the second independent variable, and it had five levels: Repeat and Nonrepeat Trials 1-10 (Epoch 1), Repeat and Nonrepeat Trials 11-20 (Epoch 2), Repeat and Nonrepeat Trials 21-30 (Epoch 3), Repeat and Nonrepeat Trials 31-40 (Epoch 4), and Repeat and Nonrepeat Trials 41-50 (Epoch 5). Once again, the dependent measure was the percentage of targets reported accurately.

\section{Results}

Target 1 accuracy for nonrepeat trials. Overall, T1 accuracy was $86.4 \%$. T1 accuracy did not significantly differ across trial epoch.

Target 1 and Target 2 accuracy. Figure 7 shows the mean T1\&T2 percentage of accuracy as a function of repetition and trial epoch for Experiment 6. Planned contrasts revealed that although there was significant repetition blindness in Trial Epochs 1 and 2 [first 40 dual-target trials; $t(1,17)=4.5, p<.0003$ ], there was no significant repetition deficit across Trial Epochs 3, 4, and 5 [last 60 dual-target trials; $t(1,17)=0.02, p=.98]$.

Intrusions. In the dual-target trials, the subjects made repetition intrusions on 11 out of the 1,700 trials pre- 


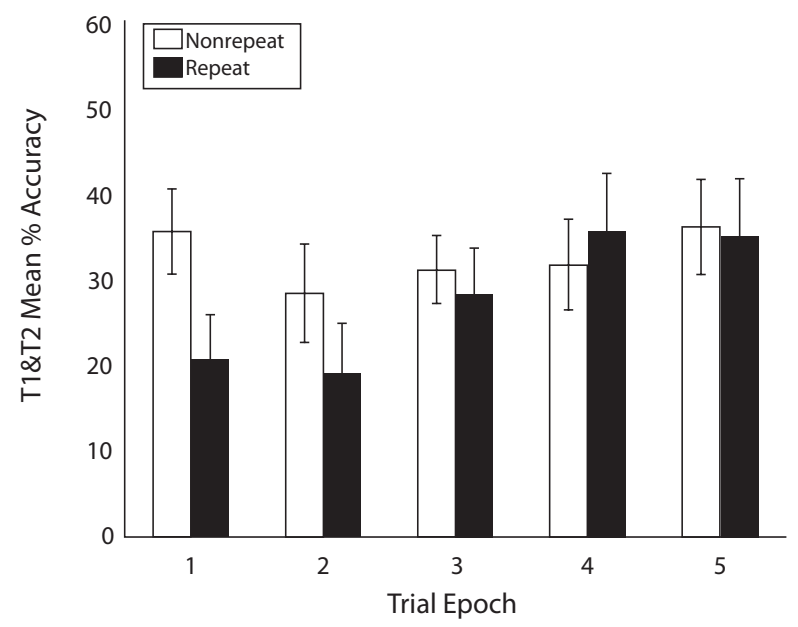

Figure 7. Mean percentage of trials in Experiment 6 where both Target 1 and Target 2 (T1\&T2) were reported correctly as a function of repetition and trial epoch.

sented, and in none of the single-target trials were repeat targets reported. Thus, a bias for guessing repetitions was not responsible for the pattern of results observed.

\section{Discussion}

Did Kanwisher (1991) find repetition blindness, in a task in which targets were defined featurally, due to the small number of trials presented to subjects? To test this here, we presented streams containing red letter targets and black letter distractors and examined the effect of repetition as a function of trial number. Across the first 40 trials, the subjects displayed significant repetition blindness; however, across the next 60 trials, there was no repetition deficit. Thus, it appears that it takes subjects time to employ featural differences between targets and distractors to enhance target registration, and this is most likely the reason why Kanwisher (1991) observed repetition blindness in her experiment (see also Chun, 1997), rather than a null effect of repetition or repetition priming. Again, the subjects made very few repetition intrusions, and the guessing correction applied to the T1\&T2 data had no effect on the pattern of results; therefore, the absence of repetition blindness at Trial Epochs 3, 4, and 5 is not due to guessing.

\section{COMPARISON OF EXPERIMENTS}

Thus far, we have demonstrated that when repeated targets in an RSVP stream are the same color as one another but differ in color from the distractors, repetition blindness is completely removed and is replaced by repetition priming. This effect is contingent on the targets' being separated by at least one distractor and on subjects' use of the featural cues to select the targets. We considered the possibility that the repetition priming observed might be due to the subjects' adopting a guessing bias of reporting repeats and assessed this by using the guessing correction introduced by Chun (1997). There was no evidence of such a bias to report repeat targets. However, below, we will consider another possible account of the increased report of repeat items.

Harris and Morris (2004) have suggested that when task difficulty is high and accuracy is low in the nonrepeat condition $(<20 \%)$, subjects treat the dual-target RSVP task more as a same/different judgment between targets than as one that requires full encoding. They speculated that subjects encode the first target and then judge whether the second is the same or different: If subjects judge T2 as being the same as $\mathrm{T} 1$ they report repeat targets; if different, subjects guess the identity of T2. Adopting this strategy may lead to superior report in the repeat condition, relative to the nonrepeat condition (repetition priming), and more important, it would not be accounted for by the Chun (1997) correction, since the subjects would guess few repeats when nonrepeat targets were presented. In many of our experiments in which repetition priming was found, nonrepeat trial accuracy was low (however, it was greater than $20 \%$ ), as compared with that observed in Experiment 1, where we found significant repetition blindness for colored targets. Thus, it is possible that the guessing bias proposed by Harris and Morris does, indeed, give rise to the repetition priming effect in our study. To assess this, we compared nonrepeat performance across our experiments where repetition blindness and repetition priming were observed. When the nonrepeat performance in the black target trials in Experiment 1 is compared with that in the digit distractor trials (red targets) of Experiment 4, using a 2 (Experiment 1 vs. Experiment 2$) \times 5$ (lag) between-subjects ANOVA, there is neither a main effect of experiment nor an interaction between experiment and lag $(F<2, p>.1)$. Thus, even though we found significant repetition blindness in the black target trials in Experiment 1 and significant repetition priming in the digit distractor trials in Experiment 4, there was no difference in nonrepeat performance between these conditions. 
Similarly, when nonrepeat performance was compared in Experiments 2 and 3 at lag 1, there was no significant difference $(F<1.8, p>.18)$. Yet, at lag 1 in Experiment 2, there was significant repetition blindness, and in Experiment 3 , significant repetition priming $\left(F_{\mathrm{s}}>7, p \mathrm{~s}<.009\right)$. Thus, these results clearly show that repetition blindness and repetition priming can occur in conditions that do not significantly differ in nonrepeat performance; thus, repetition priming in our experiments (and others; cf. Coltheart \& Langdon, 2003) was not simply due to a guessing bias.

\section{GENERAL DISCUSSION}

We have demonstrated that in an RSVP stream, where two targets differ featurally from distractors, repetition blindness does not occur and repetition enhances target report. The experiments demonstrate that this benefit of repetition is seen only when (1) the subjects use the featural cues to select the targets and (2) the targets are episodically separate from one another (i.e., identical targets are separated by at least one distractor, or targets differ featurally from one another, as well as from the distractors). These findings extend those of Chun (1997), who previously demonstrated that when targets differ featurally from the distractors, as well as from each other, repetition blindness no longer occurs, and a trend toward repetition priming is elicited. More important, they show that featural differences between targets and distractors play a vital role in the process of registering stimuli as distinct objects.

As was previously discussed, our results differ from those of Kanwisher (1991) and Campbell et al. (2002), who found repetition blindness in an RSVP task when targets and distractors differed in color. As was previously discussed, the difference between the results presented here and those of Kanwisher (1991) appears to be due to the difference in trial number. This was confirmed in Experiment 6 (see also Chun, 1997), which demonstrated that it takes subjects time to learn how to use featural differences between targets and distractors to distinguish objects. The differences between our results and those of Campbell et al. (2002) are most likely due to subjects' failing to make full use of the featural target cues in Campbell et al.'s study. In Campbell et al.'s experiment, targets were featurally defined on only half the trials, intermixed within blocks; therefore, the subjects could not simply use color to define the targets. Our data from Experiments 1 and 4 suggest that featural cues elicit repetition priming only when they are employed to select targets. Since the targets were colored only on half of the trials in Campbell et al.'s study, it is unlikely that the subjects used these featural cues to select the targets, and as a result, they did not find repetition priming.

Although it appears that featural differences between targets and distractors can lead to repetition priming, there is little evidence that category differences between stimuli influence the processing of repeated items in RSVP streams. Chun (1997) found repetition blindness when the targets were letters among digit distractors, an effect we replicated in Experiment 1. The failure to find repetition priming under these conditions suggests that categorical differences between targets and distractors do not provide episodic cues that can be used to enhance registration. In addition, repetition priming is not contingent on the targets' differing in shape, since we found this effect when the letter case of the targets was held constant.

\section{A Type-Token Account of Repetition Priming in RSVP}

Chun (1997; see also Kanwisher, 1987; Neill, Neely, Hutchison, Kahan, \& VerWys, 2002) proposed a typetoken model to account for both the attentional blink and repetition blindness he observed in his study. He suggested that the recognition of visual stimuli is undertaken by two separate processing modules, one responsible for extracting type information that pertains to the identity of a stimulus, and another that extracts token information that represents the stimulus's spatiotemporal location. The model predicts that representations of type and spatiotemporal token information are established for virtually all the stimuli presented in the RSVP stream; however, conscious percepts are generated only for those items that are the subject of focal attention and have their type and token information bound together. This process of binding type and spatiotemporal information together to form an object token is capacity limited, and it is this limitation that is responsible for both repetition blindness and the attentional blink. Repetition blindness occurs as subjects fail to bind a second spatiotemporal token to an already activated type, whereas the attentional blink results when a second type with a different identity is not consolidated (Chun, 1997).

The type-token framework accounts for the effects of repetition on dual-target RSVP streams as follows. When subjects are required to report the identity of two black letter targets that appear in a stream of black digit distractors, only type information can be used as a basis for discriminating items. As a result, at short temporal lags, the two identical stimuli are not separately registered (tokenized) and are encoded as the same object; consequently, repetition blindness is observed (Chun, 1997; Chun \& Cavanagh, 1997).

Whereas only type information can be used to individuate/register two items in an RSVP stream when the target stimuli do not differ featurally from the distractors, in trials on which targets are defined by a specific feature (e.g., color), this information can be used to mark the appearance of targets (i.e., abrupt color onsets help subjects become aware that targets have been presented). In the case of Chun's (1997) third experiment and our fourth experiment, in which a red letter target and a green letter target were presented among a stream of black letter distractors, the targets would have "popped out" and, as a result, would have undergone attentive processing. At the stage of individuation/registration, these featural differences would have prevented a repetition deficit, since the subjects had two separate visual cues that indicated the presence of two targets.

Our finding of repetition priming when two red letter targets were presented within a stream of black letter distractors can also be accounted for under the same frame- 
work: The featural difference between the targets and the distractors would have provided two episodic cues that were distinct from the distractors, but not from each other. This would have ensured that the subjects perceived two separate target stimuli.

An interesting question is why we obtained repetition blindness in our third experiment when the targets were adjacent. This could be taken as evidence against our claim that featural differences between targets and distractors enhance token individuation/registration. As has previously been discussed, however, the presentation of the two adjacent target stimuli resulted in a single red "event"; thus, there was only one color marker, and the targets were not encoded as two separate items. The results of Experiment 3 support this conclusion, since they demonstrate that repetition priming was elicited at lag 1 when the two targets were separated episodically from one another by having different colors. This suggestion is consistent with findings of Chun and Cavanagh (1997), who have shown that the tokens that subserve repetition blindness are conceptually similar to those involved in apparent motion. Apparent motion occurs when subjects perceive two separate visual events as being different states in the history of a single object in motion (Anstis, 1980). Chun and Cavanagh presented two sets of alphanumeric streams that were spatially displaced and set up to create appropriate spatiotemporal conditions for apparent motion to occur. They found that repetition blindness was greater when the targets were presented within a single apparent motion stream than when the targets appeared in different streams. These results demonstrated an increased repetition deficit when identical targets were perceived as two events in the same object's history and, thus, provided support for the type-token model.

Thus far, we have suggested that the repetition priming elicited in our experiments is due to the featural cues acting as episodic markers for attention that enhance processing of the second target on repeat trials. An explanation based only on improved encoding due to featural differences between targets and distractors could explain a null effect of repetition on target report. However, it cannot fully account for the benefit of repetition found here, since the featural cues would enhance registration equally for both repeat and nonrepeat trials. Obviously, repetition must benefit initial target recognition, because the only difference between the repeat and the nonrepeat trials is the number of identities that must be consolidated. We suggest that repetition priming occurs because initial identification of the second target is facilitated if a preceding item activates the same stored abstract letter identity.

Since the demonstrations by Scarborough, Cortese, and Scarborough (1977) of facilitatory repetition priming, faster and more accurate recognition of a repeated word than of its first occurrence, many investigations have revealed that repetition priming can occur even when the initial occurrence, the prime, is presented too briefly for conscious awareness (Forster \& Davis, 1984). However, although it may seem plausible that it is easier to identify a stimulus a second time in an RSVP stream, this suggestion has been debated in the literature. Kanwisher
(1987) used a threshold task in which she presented subjects with an RSVP stream of words and asked them to name the last stimulus, which was followed by a pattern mask. On half the trials, the target was preceded by an identical item, and on the other half, there were no repeats in the stream. Target accuracy was superior on repeat trials, when compared with nonrepeat trials, thus providing evidence that stimulus registration is facilitated when a preceding item activates the same identity representation. However, Kanwisher and Potter (1990; see also Luo \& Caramazza, 1995; Whittlesea, Dorken, \& Podrouzek, 1995) failed to replicate this effect and actually found a repetition deficit.

A problem with the threshold task, which may be the cause of this discrepancy in findings, is that when only the last item requires report, the extent to which subjects process the distractors is not controlled and may be influenced by differing strategies. For example, in Kanwisher's (1987) study, in which there were many distractors, only type activation may have occurred for the first occurrence of the repeated item, and as a result, repetition benefited target report. In Luo and Caramazza's (1995) study, however, there were only two items (the subjects having to report only the second stimulus), and therefore, it is possible that the subjects may have completely tokenized the first item, which would have resulted in a repetition deficit.

Shapiro, Driver, Ward, and Sorensen (1997) devised a task that was better able to control the extent to which subjects processed the priming stimulus and provided data that suggest that identification of a target is facilitated by a preceding stimulus with the same identity. They presented subjects with an RSVP task in which the subjects had to report three targets, T1 being defined by color and $\mathrm{T} 2$ and $\mathrm{T} 3$ by category (letters amongst digit distractors). T2 and T3 had either the same identity ( $P$ and $p$ ) or a different identity $(P$ and $d)$, and interest focused on the report accuracy for $\mathrm{T} 3$, comparing trials on which $\mathrm{T} 2$ was reported correctly and trials on which it was missed. Shapiro et al. found enhanced report of T3 on repeat trials when $\mathrm{T} 2 \mathrm{had}$ been missed and a repetition deficit for T3 when $\mathrm{T} 2$ had been correctly reported.

Thus, repetition can increase the likelihood that a second target will be reported, but in the studies mentioned above, this benefit occurred when the first occurrence of the repeat failed to be reported. We therefore propose that successful report of both items was observed in our study because the featural cues promoted type-token consolidation of both targets and, as a result, the prior occurrence of the repeated item facilitated report of the second target. To summarize, when two targets are presented with the same identity in an RSVP steam, initial identification of the second target is facilitated, but whether repetition blindness or repetition priming is observed (i.e., whether the second stimulus is or is not consciously perceived) depends on how episodically distinct the two targets are, which is influenced by both featural and temporal variables.

\section{Implications for Theories of Repetition Blindness}

The focus of the present article has been to investigate whether featural differences between targets and dis- 
tractors enhance target registration in dual-target RSVP search; however, the data also have implications for models of repetition blindness. We have offered a type-token account of our data (Chun, 1997; Kanwisher, 1987; Neill et al., 2002; Potter, 1999); however, alternative theoretical accounts have been offered to explain the processing of repeated stimuli in RSVP.

After failing to replicate Kanwisher's (1987) finding that a distractor could enhance report of an identical target in a single RSVP stream, Luo and Caramazza $(1995,1996)$ offered a perceptual account of repetition blindness. They postulated that repetition blindness is the result of type nodes' having short refractory periods that make them less sensitive to subsequent presentations of the same stimulus for a brief interval after they are initially activated. Thus, their hypothesis states that when a repeated target item appears shortly after the first target, it cannot activate the type sufficiently for it to be consciously registered.

The type refractoriness (Luo \& Caramazza, 1995, 1996) account is an attractive alternative hypothesis to token individuation models (e.g., Chun, 1997; Kanwisher, 1987). Not only does it offer a parsimonious explanation of repetition deficits, but also it fits well with research in the field of neurophysiology that has demonstrated that neurons have a period in which their excitability is reduced after initial firing. Although the type refractoriness model appears to be a viable account of repetition blindness, it does have difficulty explaining the following findings. The fact that repetition blindness is increased when repeated stimuli appear in the same apparent motion stream (Chun \& Cavanagh, 1997) and is removed when two differently colored targets are presented among a stream of black distractors (Chun, 1997) is evidence against this model. Both the apparent motion and the color manipulations influence the ease with which registration/tokenization occurs and have no effect on type activation; therefore, it is unclear how the refractory period of the types can explain these results. The priming study of Shapiro et al. (1997) discussed above also provides evidence that is counter to the predictions of the type refractoriness model. Finally, the results from our study are also inconsistent with the type refractoriness account. In our experiments, the two featurally defined repeated targets would have activated the same stored letter representation; thus, if a type refractoriness mechanism were responsible for repetition blindness, one would expect to find a repetition deficit, rather than the repetition priming effect obtained in Experiments 2-6.

Alternatively, several researchers have proposed that repetition blindness is not the result of an initial encoding difficulty but, rather, that the effect has its locus at later stages of processing (e.g., Armstrong \& Mewhort, 1995; Fagot \& Pashler, 1995). These accounts differ but assume both that repeated targets appearing in an RSVP stream are registered in memory and that the deficit arises at the stage of retrieval. Memory retrieval accounts were motivated by the fact that, originally, sentence and list recall were used to examine repetition blindness and such tasks are clearly influenced by retrieval processes. The paradigm used in this study required subjects to report only two items, and thus it is unlikely to be affected by retrieval limitations.
Furthermore, featural distinctiveness of targets led to repetition priming instead of repetition blindness. Therefore, it appears that there is not a general bias against reporting repetitions that appear in an RSVP stream.

We found significant repetition priming at lags 2 and 3 in Experiment 2 but observed repetition blindness at lag 1 . We have argued that a token individuation account would predict the occurrence of repetition blindness at lag 1 , since the adjacent repeated items are likely to be encoded as a single event in the absence of two separate color cues to promote consolidation. This pattern of results is the reverse of that found with short lists presented at the longer stimulus onset asynchrony of $500 \mathrm{msec}$ between items. Henson (1998) obtained repetition facilitation for adjacent repeated letters and found a repetition deficit at the lags that yielded repetition priming for items shown at RSVP rates in our experiments. He likewise concluded that different mechanisms underlie the repetition effects arising at these vastly differing exposure durations.

In addition, it has been demonstrated that repetition blindness occurs when memory demands are reduced (Chun, 1997; Dux \& Marois, 2007; Luo \& Caramazza, 1995, 1996) and even when no recall is required (Hochhaus \& Johnston, 1996; Johnston et al., 2002). Thus, although retrieval failure contributes to some effects of repetition seen in RSVP streams, it is not responsible for all of them.

A final theory of repetition blindness to be discussed is the construction and attribution account of Whittlesea and colleagues (Masson, 2004; Whittlesea et al., 1995; Whittlsea \& Masson, 2005). This hypothesis attributes repetition blindness to subjects' being unable to encode the context of the repeat stimuli, and as a result, when the RSVP stimuli are reconstructed for conscious report, the subjects are unable to identify the repeat stimuli as being separate. The construction and attribution account and the type-token theory make many of the same predictions regarding the effect that increasing the distinctiveness of targets should have on repetition blindness. Thus, the present data support the construction and attribution account to some extent. One problem for this hypothesis, however, is the repetition blindness found at lag 1 in Experiment 2. In this condition, the subjects had to attend only to two items in the RSVP stream, as they did at the other lags. So why, then, was the context of the targets more impoverished than at lags 2 and 3 ? The finding that repetition blindness occurred when the red targets appeared next to one another in the RSVP stream and was completely removed when they were separated by one or more distractor items suggests that the extent to which repeat targets are perceived as the same object has a strong influence on repetition blindness - a prediction of the type-token model, but not of the construction attribution account.

\section{Conclusion}

The presentation, in an RSVP stream, of two identical targets that differ featurally from the distractors can lead to repetition priming. Previous work has shown that categorical differences between targets and distractors do not remove repetition blindness; therefore, it appears that featural differences, rather than just general distinctive- 
ness, provide effective visual cues to attract attention and enhance the perception of the targets. These data support a type-token (Chun, 1997; Kanwisher, 1987; Neill et al., 2002) account of visual processing and suggest that when establishing the presence of two temporally separated items in the environment, not only is it important how they differ featurally from one another, but also how they differ from other stimuli in close temporal proximity.

\section{AUTHOR NOTE}

Correspondence concerning this article should be addressed to P. E. Dux, Department of Psychology, Vanderbilt University, 428 Wilson Hall, 111 21st Ave. So., Nashville, TN 37203 (e-mail: paul.dux@vanderbilt .edu).

\section{REFERENCES}

Anstis, S. M. (1980). The perception of apparent movement. Philosophical Transactions of the Royal Society B, 290, 153-168.

Armstrong, I. T., \& Mewhort, D. J. K. (1995). Repetition deficit in rapid-serial-visual-presentation displays: Encoding failure or retrieval failure? Journal of Experimental Psychology: Human Perception \& Performance, 21, 1044-1052.

Brainard, D. H. (1997). The Psychophysics Toolbox. Spatial Vision, 10, 433-436.

Broadbent, D. E., \& Broadbent, M. H. P. (1987). From detection to identification: Response to multiple targets in rapid serial visual presentation. Perception \& Psychophysics, 42, 105-113.

Campbell, J. I. D., Fugelsang, J. A., \& Hernberg, V. D. (2002). Effects of lexicality and distinctiveness on repetition blindness. Journal of Experimental Psychology: Human Perception \& Performance, 28, 948-962.

Chun, M. M. (1997). Types and tokens in visual processing: A double dissociation between the attentional blink and repetition blindness. Journal of Experimental Psychology: Human Perception \& Performance, 23, 738-755.

Chun, M. M., \& CaVAnagh, P. (1997). Seeing two as one: Linking apparent motion and repetition blindness. Psychological Science, 8, 74-79.

Coltheart, V., \& LANGdon, R. (2003). Repetition blindness for words yet repetition advantage for nonwords. Journal of Experimental Psychology: Learning, Memory, \& Cognition, 29, 171-185.

Dux, P. E., \& Coltheart, V. (2005). The meaning of the mask matters: Evidence of conceptual interference in the attentional blink. Psychological Science, 16, 775-779.

Dux, P. E., \& MARoIs, R. (2007). Repetition blindness is immune to the central bottleneck. Psychonomic Bulletin \& Review, 14, 729-734.

Fagot, C., \& Pashler, H. (1995). Repetition blindness: Perception or memory failure? Journal of Experimental Psychology: Human Perception \& Performance, 21, 275-292.

Forster, K. I., \& Davis, C. (1984). Repetition priming and frequency attenuation in lexical access. Journal of Experimental Psychology: Learning, Memory, \& Cognition, 10, 680-690.

Forster, K. I., \& Forster, J. C. (2003). DMDX: A Windows display program with millisecond accuracy. Behavior Research Methods, Instruments, \& Computers, 35, 116-124.

Harris, C. L., \& Morris, A. L. (2004). Repetition blindness occurs in nonwords. Journal of Experimental Psychology: Human Perception \& Performance, 30, 305-318.

HENSON, R. N. A. (1998). Item repetition in short-term memory: Ranschburg repeated. Journal of Experimental Psychology: Human Perception \& Performance, 24, 1162-1181.

Hochraus, L., \& Johnston, J. C. (1996). Perceptual repetition blindness effects. Journal of Experimental Psychology: Human Perception $\&$ Performance, 22, 355-366.

Johnston, J. C., Hochhaus, L., \& Ruthruff, E. (2002). Repetition blindness has a perceptual locus: Evidence from online processing of targets in RSVP streams. Journal of Experimental Psychology: Human Perception \& Performance, 28, 477-489.

Kanwisher, N. G. (1987). Repetition blindness: Type recognition without token individuation. Cognition, 27, 117-143.

KANWISHER, N. [G.] (1991). Repetition blindness and illusory conjunc- tions: Errors in binding visual types with visual tokens. Journal of Experimental Psychology: Human Perception \& Performance, 17, 404-421.

Kanwisher, N. G., \& Potter, M. C. (1990). Repetition blindness: Levels of processing. Journal of Experimental Psychology: Human Perception \& Performance, 16, 30-47.

Luo, C. R., \& CARAmazza, A. (1995). Repetition blindness under minimum memory load: Effects of spatial and temporal proximity and the encoding effectiveness of the first item. Perception \& Psychophysics, 57, 1053-1064.

Luo, C. R., \& Caramazza, A. (1996). Temporal and spatial repetition blindness: Effects of presentation mode and repetition lag on the perception of repeated items. Journal of Experimental Psychology: Human Perception \& Performance, 22, 95-113.

Masson, M. E. J. (2004). When words collide: Facilitation and interference in report of words from rapidly presented lists. Journal of Experimental Psychology: Learning, Memory, \& Cognition, 30, 1279-1289.

Neill, W. T., Neely, J. H., Hutchison, K. A., Kahan, T. A., \& VerWys, C. A. (2002). Repetition blindness, forward and backward. Journal of Experimental Psychology: Human Perception \& Performance, 28, 137-149.

Pelli, D. G. (1997). The VideoToolbox software for visual psychophysics: Transforming numbers into movies. Spatial Vision, 10, 437-442.

Potter, M. C. (1999). Understanding sentences and scenes: The role of conceptual short-term memory. In V. Coltheart (Ed.), Fleeting memories: Cognition of brief visual stimuli (pp. 13-46). Cambridge, MA: MIT Press.

PotTer, M. C., \& LeVy, E. I. (1969). Recognition memory for a rapid sequence of pictures. Journal of Experimental Psychology, 81, 10-15.

Raymond, J. E., Shapiro, K. L., \& Arnell, K. M. (1992). Temporary suppression of visual processing in an RSVP task: An attentional blink? Journal of Experimental Psychology: Human Perception \& Performance, 18, 849-860.

Scarborough, D. L., Cortese, C., \& Scarborough, H. (1977). Frequency and repetition effects in lexical memory. Journal of Experimental Psychology: Human Perception \& Performance, 3, 1-17.

Shapiro, K. L., Driver, J., WARD, R., \& Sorensen, R. E. (1997). Priming from the attentional blink: A failure to extract visual tokens but not visual types. Psychological Science, 8, 95-100.

Visser, T. A. W., Bischof, W. F., \& Di Lollo, V. (1999). Attention switching in spatial and nonspatial domains: Evidence from the attentional blink. Psychological Bulletin, 125, 458-469.

Weichselgartner, E., \& Sperling, G. (1987). Dynamics of automatic and controlled visual attention. Science, 238, 778-780.

Whittlesea, B. W. A., Dorken, M. D., \& Podrouzek, K. W. (1995). Repeated events in rapid lists: Part I. Encoding and representation. Journal of Experimental Psychology: Learning, Memory, \& Cognition, 21, 1670-1688.

Whittlesea, B. W. A., \& Masson, M. E. J. (2005). Repetition blindness in rapid lists: Activation and inhibition versus construction and attribution. Journal of Experimental Psychology: Learning, Memory, \& Cognition, 31, 54-67.

\section{NOTES}

1. Although we refer to manipulating the color of the stimuli in this study, it should be noted that color and luminance were confounded here. This has also been the case in previous studies in which the effect of coloring targets on repetition blindness has been examined.

2. We replicated the results found at lags 2 and 3 of this experiment in a separate experiment $(n=18)$ in which the stimuli were all uppercase letters and the targets were defined by color (red targets among black distractors). There was an overall main effect of repetition in this experiment $[F(1,17)=5.3, p<.05]$, with better target report on repeat trials $(36.7 \%)$ than on nonrepeat trials $(28.1 \%)$. In addition, there was also a significant effect of lag $[F(4,68)=28.7, p<.0002]$, revealing the standard attentional blink pattern.

(Manuscript received March 20, 2007; revision accepted for publication November 26, 2007.) 